\title{
Safety and feasibility of same-day discharge laparoscopic radical prostatectomy: a systematic review
}

\author{
Amine Saouli, ${ }^{1,2} \cdot$ Razvan-George Rahota $^{3} \cdot$ Imad Ziouziou $^{4} \cdot$ Othmane Elhouadfi, ${ }^{1,2} \cdot$ Tarik Karmouni $^{1,2}$. \\ Khalid Elkhader $^{1,2} \cdot$ Abdellatif Koutani $^{1,2} \cdot$ Ahmed Iben Attya Andalousi $^{1,2} \cdot$ Guillaume Ploussard $^{3}$
}

Received: 27 April 2021 / Accepted: 21 January 2022 / Published online: 14 February 2022

(c) The Author(s), under exclusive licence to Springer-Verlag GmbH Germany, part of Springer Nature 2022

\begin{abstract}
Purpose Day case or same-day discharge (SDD) pure laparoscopic or robot-assisted radical prostatectomy (RP) has risen over the last few years with the aim of discharging patients within $24 \mathrm{~h}$, reducing costs and length of stay, and facilitating return to active life. We perform a systematic review of literature to evaluate the feasibility of SDD RP.

Methods A systematic review search was performed and the following bibliographic databases were accessed: PubMed, Science Direct, Scopus, and Embase. This was carried out in accordance with the Preferred Reporting Items for Systematic reviews and Meta-analyses (PRISMA) guidelines.

Results Based on the literature search of 509 articles, 12 (1378 patients) met the inclusion criteria (mean age: 63 years). All studies were unicentric except one. The mean SDD surgeries experience per centre was 66 cases. The means operative time and blood loss were $154 \mathrm{~min}$ and $126.5 \mathrm{ml}$, respectively. Mean SDD failure was 7.4\%. Concomitant lymph node dissection was performed in $56.2 \%$. The overall complication rate was $10.2 \%$ of cases; with a majority of Clavien grade I or II. Mean readmission rate after discharge was $5 \%$. SDD generated cost reductions compared to inpatient surgery with variable differences according to the considered healthcare system.

Conclusions Day-case RP is a safe and feasible strategy in selected cases with multicentre proofs of concept. Its widespread use in routine practice needs further research due to biases in patient selection. Implementation of peri-operative pathways such as ERAS and prehabilitation improves patient adherence to SDD.
\end{abstract}

Keywords Radical prostatectomy $\cdot$ Day case $\cdot$ Same day discharge $\cdot$ Outpatient $\cdot$ Ambulatory $\cdot$ Safety

\section{Introduction}

Same day discharge (SDD) radical prostatectomy (RP) refers to patients being discharged the same day of the procedure. SDD has been suggested to be feasible in various countries. The question of outpatient surgery in radical prostatectomy

Amine Saouli

Amine.saouli0@gmail.com

1 Department of Urology B, Ibn Sina Hospital, CHU Ibn Sina, Rabat, Morocco

2 Faculty of Medicine and Pharmacy, Mohammed V University, Souissi, Rabat, Morocco

3 Department of Urology, La Croix du Sud Hospital, Quint Fonsegrives, France

4 Department of Urology, University Hospital of Agadir, Agadir, Morocco arose before the robot era by Palmer and Ruiz by perineal approach $[1,2]$.

The advent of laparoscopy and robotic assistance has led to a significant reduction in length of stay and to wider consideration of SDD RP. To date, robot-assisted radical prostatectomy (RP) is currently the preferred surgical approach for prostate cancer surgery [3]. Given its peri-operative advantages, minimally invasive surgery with robotic assistance improves patient recovery and length of stay compared with open procedure.

The first prospective study of ambulatory laparoscopic radical prostatectomy was published by Martin in 2010 [4]. Since then, there are growing reports on the safety and feasibility of performing minimally invasive urologic oncology surgeries in the outpatient setting $[3,5]$.

However, outcomes of outpatient RARP have been described in very few reports which are mostly based on single institution experiences except for a recent, French, 
multicentric study reported by Ploussard et al. [6] While an increasing number of original studies are reporting outcomes on SDD RP, also referred to as day case or outpatient RP, critical evaluation remains under-reported as well as homogeneity between inclusion criteria.

SDD RP is also likely to have increased interest given current healthcare issues regarding the COVID-19 pandemic and providing context for why clinicians would be interested in same-day discharge (cost reduction, length of stay, patient satisfaction, etc.).

The aim of this study was to evaluate the safety and the feasibility of routine outpatient management after radical prostatectomy (RP).

\section{Methodology}

A priori protocol was submitted to PROSPERO for registration on September 2020. The PROSPERO registration number was: CRD42020185180.

The scope of the review according to PICO is as follows: $\mathrm{P}$ - population consisted of patients with prostate cancer; I-who underwent RP with outpatient surgery; C-or inpatient surgery; $\mathrm{O}-\mathrm{Outcomes}$ of interest were perioperative outcomes.

The data analysed included: sample size, age, BMI, ASA, PSA, TNM stage, Gleason score, type of surgery, rate and severity of postoperative complications according to the Clavien-Dindo classification.

\section{Inclusion/exclusion criteria}

Study inclusion criteria were as follows: (i) Studies with the objective to discharge patients the same day after RP; (ii) patients treated with laparoscopic or robot-assisted RP; (iii) year of publication 2000-2020.

Exclusion criteria for considering SDD were different according to studies. However, main consensus factors were: (i) Oral anticoagulation; (ii) alone at home at discharge; (iii) long distance from home to hospital (varying cut-off: 50-150 kms); (iv) patient preference. The data analysed included: sample size, inclusion criteria (age, BMI, ASA, PSA, TNM stage, Gleason score), type of surgery, rate and severity of postoperative complications according to the Clavien-Dindo classification.

\section{Study design}

The authors performed a systematic review of the world literature to identify any original studies performed where adult patients underwent RP with the objective to discharge patients at day 0 . This was carried out in a Cochrane style and in accordance with the Preferred Reporting Items for
Systematic Reviews and Meta-analyses (PRISMA) checklist. Full-text publications using Roman alphabet were considered. SDD RP was defined as discharge of patients at postoperative day 0 (Fig. 1).

\section{Search strategy}

We performed a systematic literature search in three databases (PubMed/Medline, Embase and Science Direct) with the search query: "(radical prostatectomy) OR same-day discharge OR ambulatory OR day case OR outpatient)". Case reports were excluded. Individual urological journals, conference proceedings, and citation lists were also hand searched.

\section{Data extraction and synthesis}

As for the search process, data extraction was carried out by two authors (A.S) and (I.Z). Primary outcomes of interest were readmission rate and complications. Adverse events were graded according to the Clavien-Dindo classification. The grading had already been assigned by each of the individual studies. Secondary outcomes of interest included blood loss, hospital stay, and operative time. Given the low number of selected studies, no exclusion was performed based on the retrospective nature or the number of patients.

\section{Risk of bias or quality assessment}

Risk of bias was assessed according to EAU recommendations for performing systematic reviews and meta-analysis [7]. The Quality Appraisal tool for case series using a Modified Delphi technique was used for retrospective studies [8].

We assessed the risk of bias in non-randomized trials using the ROBINS-I tool in material supplementary.

\section{Results}

From a total of 509 articles, 12 studies were selected, which satisfied our predefined search criteria [4, 6, 9-18]. These included six prospective and six retrospective studies published between 2010 and 2020. The overall number of SDD per study ranged between 9 and 358 patients.

\section{Baseline characteristics}

Across the 12 studies, a total of 1189 patients (mean age 63 year; range 56.6-65.8 years) underwent laparoscopic RP. Population characteristics are listed in Table 1. Robot assistance was used in all except one study [11]. Mean BMI and PSA were $26.9 \mathrm{~kg} / \mathrm{m}^{2}$ (range 25-30), and $8.0 \mathrm{ng} / \mathrm{ml}$ (range 5.7-9.5) respectively. Mean prostate volume was $47.6 \mathrm{ml}$. 

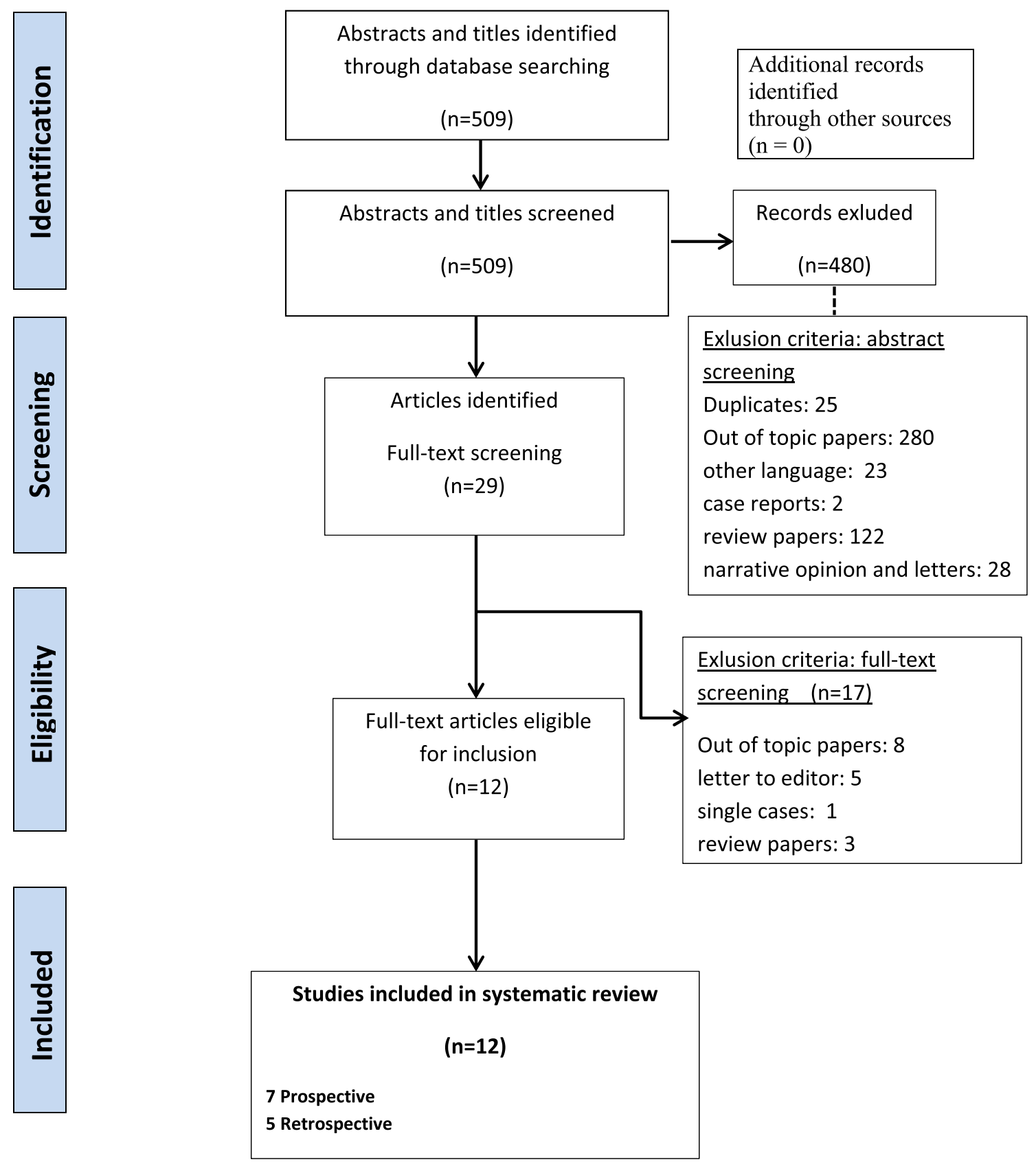

Fig. 1 Systematic review PRISMA flow diagram

High grade (ISUP 3 or more) was reported in $14-59 \%$ and SDD was considered whatever the risk classification.

\section{Outcomes of interest}

Intra-operative features are showed in Table 2. Mean operative time and blood loss were $138 \mathrm{~min}$ (range 77-198 $\mathrm{min}$ ), and $137 \mathrm{ml}$ (range 53-228 ml) respectively. Concomitant lymph node dissection was performed in $56.2 \%$ of patients, including three studies with lymph node dissection in all
SDD patients [15, 16, 18]. Lymph node dissection was not performed in one study [12]. Nerve-sparing surgery was performed in 53\% of cases (from 26 to $93 \%$ ).

Catheter removal was planned between 5 and 14 days with a removal mainly scheduled at day 7 . A drain was put intraoperatively in two studies and removed at day 0 in the majority of cases. Surgical margins were positive in $18.4 \%$ (range 3.9-28.0). A pT3-4 stage was reported in 9.0-55.5\% of cases with a rate of $\mathrm{pN} 1$ disease ranging from 2.2 to $11.0 \%$. 


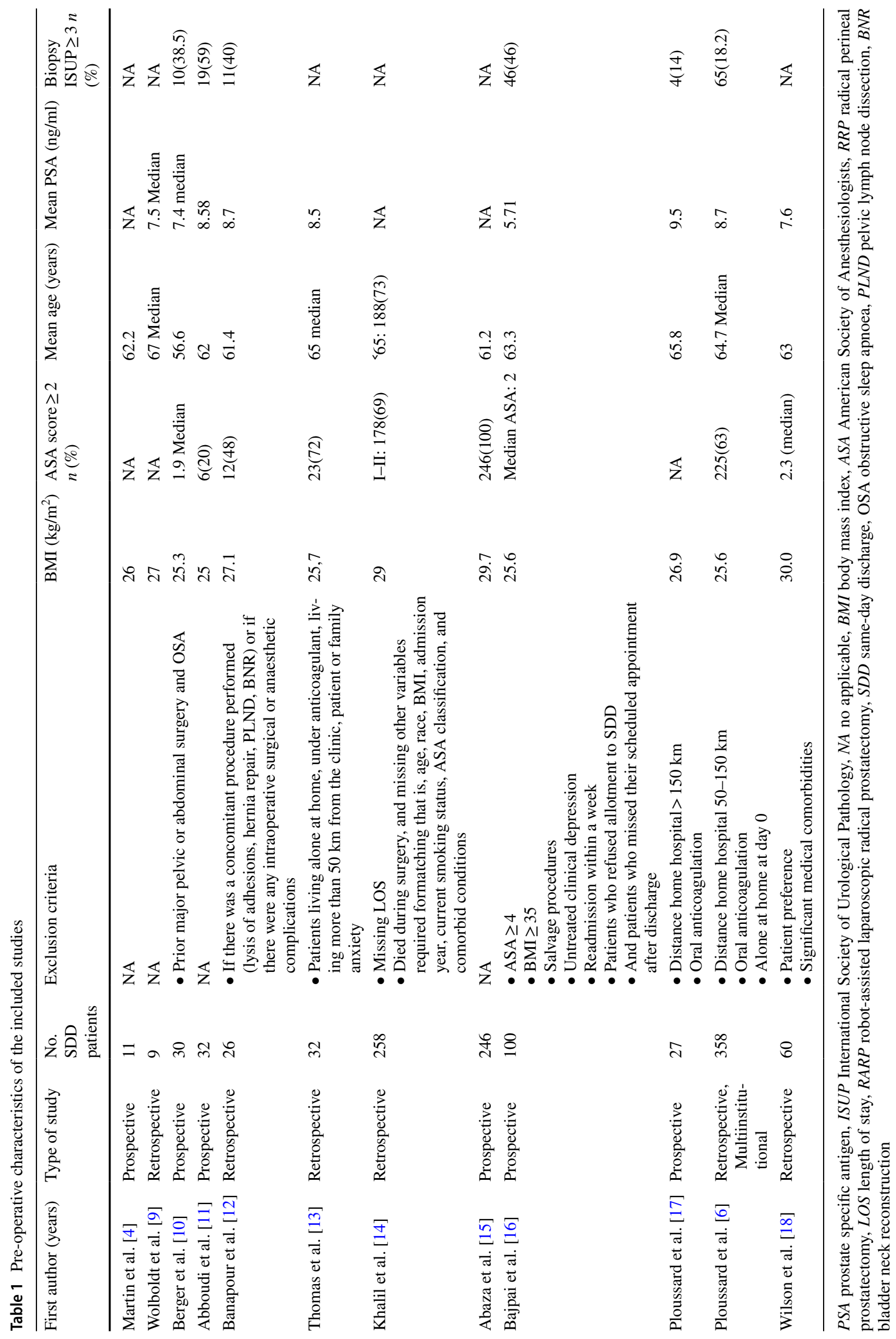




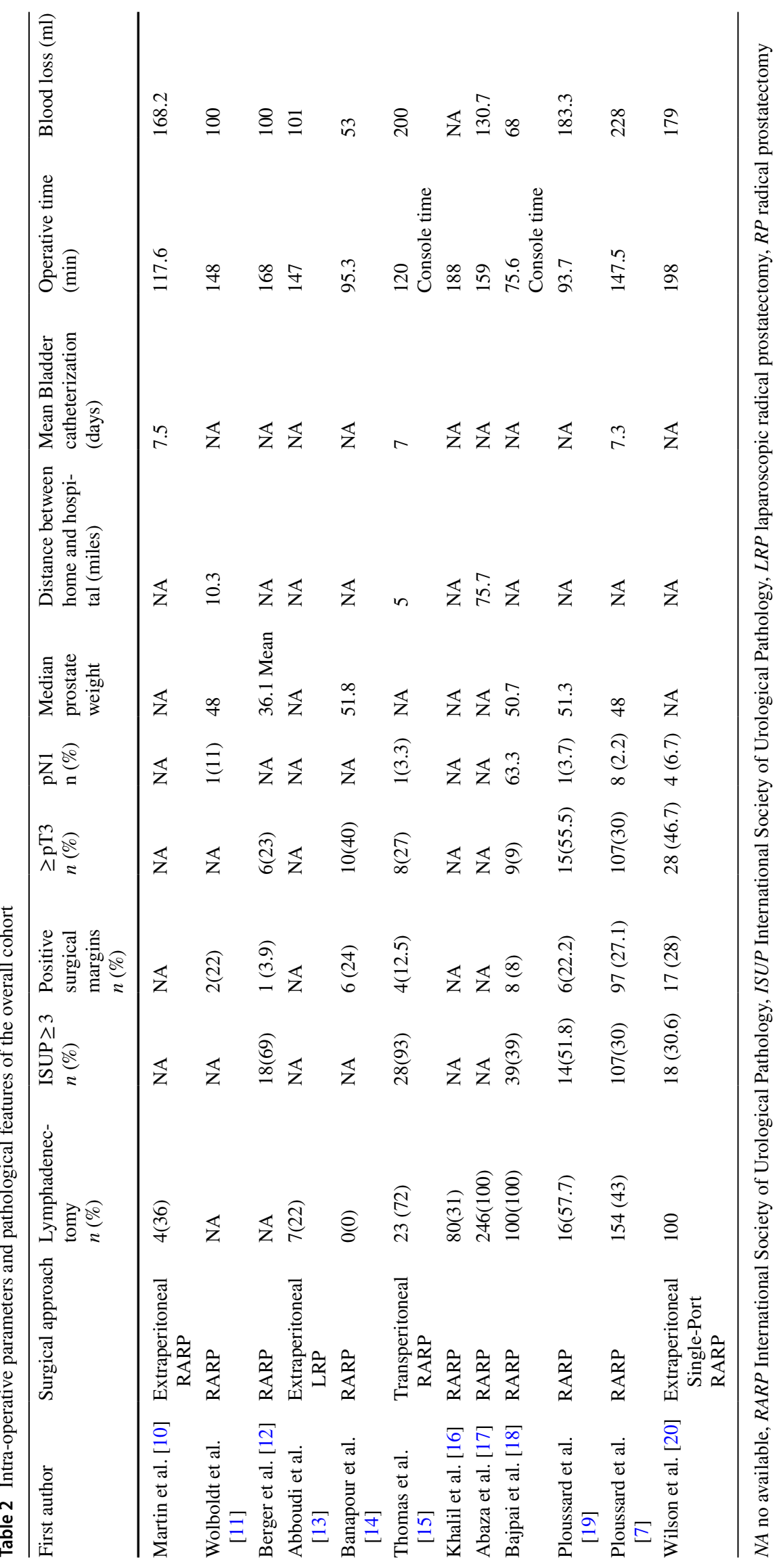


Post-operative course was described in Table 3. The mean SDD failure was $7.4 \%$, ranging from 0 to $16.7 \%$ among studies. An ERAS pathway was applied to SDD patients in 5 studies. Most studies reported a low mean pain score at discharge (visual analogue scale: median 2).

\section{Complications}

The overall readmission rate was $5.0 \%$, ranging from 0 to $30 \%$. The delay between SDD and readmission was approximately five days $[6,14]$. The mean SDD failure was $7.4 \%$ for patients requiring overnight or more observation. The overall complication rate was $10.2 \%$. The majority of these complications were low grade (Clavien I or II). No grade V complication occurred. Grade I to II complication rate varied between 0 and $30 \%$. The rate of grade III complication ranged from 0 to $11.7 \%$ with a majority of symptomatic lymphoceles.

Six series have compared SDD with inpatient RP [10, 12, 14-17].

All comparisons were retrospective with or without match-paired analysis. No randomization was performed. All studies suggested that post-operative course was not negatively impacted by SDD and the shortening of length of stay, in terms of complication or readmission rates. SDD was not associated with poorer postoperative outcomes.

Table 3 Post-operative outcomes and primary endpoints: same-day discharge (SDD) surgery failure, complications, readmission

\begin{tabular}{|c|c|c|c|c|c|c|c|c|}
\hline First author & $\begin{array}{l}\text { SDD failure } \\
n(\%)\end{array}$ & ERAS & Pain score $n(\%)$ & $\begin{array}{l}\text { Readmis- } \\
\text { sion rate } n \\
(\%)\end{array}$ & $\begin{array}{l}\text { Discharge at } \\
\text { D0 includ- } \\
\text { ing inpatient } \\
\text { and outpatient } \\
\text { patients } \\
n(\%)\end{array}$ & $\begin{array}{l}\text { Delay between } \\
\text { surgery and } \\
\text { readmission } \\
\text { (days) }\end{array}$ & $\begin{array}{l}\text { Follow-up } \\
\text { (months) }\end{array}$ & Clavien* $n(\%)$ \\
\hline $\begin{array}{l}\text { Martin et al. } \\
{[10]}\end{array}$ & $0(0)$ & No & $\begin{array}{l}\text { 5(45) Pain } \\
\text { control }\end{array}$ & $0(0)$ & 11(100) & NA & 7 days & 3a: 1 (9) \\
\hline $\begin{array}{l}\text { Wolboldt et al. } \\
\text { [11] }\end{array}$ & $1(12)$ & No & NA & $0(0)$ & $9(82)$ & NA & 255 days & $0(0)$ \\
\hline $\begin{array}{l}\text { Berger et al. } \\
{[12]}\end{array}$ & $4(13)$ & No & $1(3.8)$ & $0(0)$ & $26(87)$ & NA & 11.65 & $1(3.9)$ \\
\hline $\begin{array}{l}\text { Abboudi et al. } \\
\text { [13] }\end{array}$ & $0(0)$ & No & $\begin{array}{l}3 / 5: 1(3) \\
4 / 5: 1(3)\end{array}$ & $4(13)$ & $32(100)$ & NA & 46 & $\begin{array}{l}\text { 2: } 3(9) \\
3 \mathrm{a}: 3(9)\end{array}$ \\
\hline $\begin{array}{l}\text { Banapour et al. } \\
\text { [14] }\end{array}$ & $1(4)$ & Yes & NA & $0(0)$ & NA & NA & NA & 2: 1(4) \\
\hline $\begin{array}{l}\text { Thomas et al. } \\
\text { [15] }\end{array}$ & $4(13)$ & No & $\begin{array}{l}\text { Median EVA } \\
\text { H8: } 2(6)\end{array}$ & $9(30)$ & 30(94) & NA & 9 & $\begin{array}{l}1 \text { et } 2: 8(26.7) \\
4: 1(3.3)\end{array}$ \\
\hline Khalil et al. [16] & NA & No & NA & $6(2.6)$ & NA & 4.7 & $\leq 1$ & $8(3.1)$ \\
\hline Abaza et al. [17] & NA & No & $\begin{array}{l}\text { 1/1st 3: } 3.08 \\
(0-10) \\
2 / 3-9: 1.54 \\
(0-8) \\
3 / 9-12: 0.3 \\
(0-8)\end{array}$ & $1(0.4)$ & 169(70) & $0-7$ & 3 & $\begin{array}{l}11(4.4) \\
1: 6(2.4) \\
2: 3(1.2) \\
3: 2(0.8)\end{array}$ \\
\hline Bajpai et al. [18] & NA & Yes & $\begin{array}{l}\text { Median BPI } \\
\text { pain severity } \\
\text { score: } 2(2)\end{array}$ & $0(0)$ & NA & NA & NA & $1: 4(4)$ \\
\hline $\begin{array}{l}\text { Ploussard et al. } \\
\text { [19] }\end{array}$ & $\begin{array}{l}\text { 1(3.7) } \\
\text { (SDD group) }\end{array}$ & Yes & $2(8)$ & $1(3.7)$ & $27(52)$ & NA & 8.4 & $\begin{array}{l}3(11.1) \\
2: 2(7.4) \\
3: 1(3.7)\end{array}$ \\
\hline $\begin{array}{l}\text { Ploussard et al. } \\
\text { [7] }\end{array}$ & $15(4.2)$ & Yes & $>2: 33(9.2)$ & $10(2.8)$ & 343(95.8) & 5.4 & 15 & $\begin{array}{l}60(16.8) \\
1: 40(11.2) \\
2: 15(4.2) \\
3 b: 4(1.2) \\
4: 1(0.3)\end{array}$ \\
\hline $\begin{array}{l}\text { Wilson et al. } \\
\text { [20] }\end{array}$ & $\begin{array}{l}6(12) \\
\text { (54 patients) }\end{array}$ & Yes & $\begin{array}{l}2 \\
0-1: 37 \%\end{array}$ & $4(7)$ & $45(75)$ & NA & NA & $\begin{array}{l}11(18.3) \\
3 \mathrm{a}: 7(11.7)\end{array}$ \\
\hline
\end{tabular}

$S D D$ same-day discharge, VAS Visual Analogue Scale, BPI Brief Pain Inventory, NA no available, ERAS Enhanced Recovery after Surgery 


\section{Discussion}

Given its peri-operative advantages, minimally invasive surgery with robotic assistance improves patient recovery and length of stay compared with open procedure. Thus, hospital stays after radical prostatectomy has shortened dramatically over the years. This has been achieved by the wide acceptance of minimally invasive procedure and of robotic assistance as well as continuous improvements of peri-operative management, including ERAS and prehabilitation pathways [19].

This led to the development of SDD. However, initial feasibility studies outside the era of ERAS were not in favour of routine discharge at day 0 [20].

Dobbs et al. [21] demonstrated that two-thirds of patients surveyed following RARP did not feel ready to be discharged on the day of their surgery. While ambulatory RP may offer advantages for appropriately selected candidates, it cannot be recommended systematically for all patients. The potential benefits of early discharge were, therefore, outweighed by morbidity risk in this large cohort of patients. The optimization of perioperative pathways plays a fundamental role in SDD adoption. It has been showed that his main barriers for SDD were pain control, catheter discomfort, insufficient education about postoperative care, and post-operative nausea prevention.

In an Australian study including men undergoing RARP, findings showed that early mobilization and return to work were major priorities for patients [22]. Thus, satisfaction after RARP is high, and not only determined by objective outcomes like PSA, continence, sexual function, but also by personal perception and health-related qualityof-life including a rapid return to normal functional and to work. Reducing the length of stay and promoting SDD surgery could improve the patient perception of the surgery and could shorten the return to an active, normal, personal and professional life.

However, since its early development, SDD has demonstrated safety and secondary benefits with reduced costs, comparable peri-operative outcomes, and a potential improvement regarding post-operative functional recovery after SDD compared with overnight surgery [10, 17].

This article is the first systematic review on this topic, and it globally confirms the safe implementation of an ambulatory approach for RP in carefully selected patients. In the present multi-institutional study, SDD failure, complication and readmission rates were low, demonstrating the safety of SDD in the study population. No patient experienced major intraoperative events which could contra-indicate SDD. The readmission rate was even lower than that reported in nationwide, population-based studies
[23]. Most of complications were low-grade complications, managed without re-intervention or readmission.

The main concern limiting a wider acceptance of SDD RP remains the patient selection. Patient preference was often chosen as inclusion criterion leading to an inherent bias by selecting highly motivated patients for SDD. Indeed, patients selected for outpatient RARP tended to be healthier as a majority were young ( $<65$ years), current non-smokers, low ASA class (class $\leq$ II) and no obese $(\mathrm{BMI}<30)$. Moreover, some comparative studies reported that SDD patients had shorter operating time and were less likely to receive concomitant pelvic lymph node dissection during surgery [14]. However, at least 3 studies included in this review have included SDD patients undergoing systematic lymph node dissection $[15,16,18]$. In some studies, patients eligible for SDD should have no prior major pelvic or abdominal surgery.

Recently, a multi-institutional study evaluated factors correlated with SDD failure, complications and readmission [6]. It has been found that blood loss, lymph node dissection, and pain VAS at discharge were significantly correlated with SDD failure. SDD failure was reported in $7.8 \%$ of patients with pelvic lymph node dissection compared with only $1.5 \%$ of patients who did not undergo lymph node dissection. Lymph node dissection implied a longer operative time, a wider dissection, a longer exposure to pneumoperitoneum and all these parameters may increase the risk of post-operative pain. Pain relief was also correlated with the risk of subsequent readmission. These factors might be interesting to be taken into account for SDD patient selection and counselling.

This review also showed the great heterogeneity of patient selection and perioperative outcomes among centres. Preand post-operative management may highly vary among centres regarding of ERAS, prehabilitation and follow-up protocols and impact on SDD outcomes. However, this review suggests that SDD was feasible at a national level $[6,14]$.

The preparation of the patient and of the surgery team is of grel importance for a routine adoption of SDD RP. Several reports suggested that, in addition to well-established factors for improved outcomes such as surgeon and centre volumes, patient education is critical to the adoption of SDD after RP. Ploussard et al. recently demonstrated that the implementation of prehabilitation pathways increased the proportion of SDD surgeries [24]. Prehabilitation and ERAS protocol have a synergistic effect for improving post-operative outcomes and for reducing costs after RP [19]. The benefits from fasttrack have been well assessed in general surgery [25-27]. This leads to faster recovery, improved bowel function, shorter hospital stay without increasing major complication or readmission rates compared to standard perioperative care. As prostate cancer surgery is at lower risk of severe morbidity and complications compared with colorectal or 
bladder cancer surgery, few series have assessed the ERAS advantages around RARP. Thus, the ERAS society does not provide specific guidelines around prostate cancer surgery. The International Association for Ambulatory Surgery does not specify the types of surgery apt for SDD [28].

Surgery-associated psycho-emotional stresses especially anxiety and individual pain tolerance are capable of confounding the QoL assessment postoperatively SDD discerned significantly lesser pain and interference with general activities, along with better emotional well-being (EWB) and overall general health perception than NDD [16]. The impact of patient psychology remains weakly assessed in all these feasibility studies. The studies identified in this review supports practice of ambulatory RP in carefully selected patients whereas some feasibility studies without perioperative pathways adaptation to SDD did not [20,21]. In spite of the SDD implantation, a not negligible proportion of anxious patients would not feel comfortable with the idea of SDD. Given that SDD has not yet demonstrated superiority over overnight stay (in terms of infection, complication, return to work...), it seems reasonable to let the patient self-select the type of hospital stay after presenting SDD as being safe and feasible.

From an economic perspective, SDD have been suggested to reduce the overall cost per patient with no increased cost due to emergency visits or readmissions $[15,17]$. However, to date, none of the studies undertook formal cost-effective analyses including return to active life and costs not related to direct care. Medico-economic constraints depend on the country and nation-based assessment should be considered.

Adoption of SDD RP should ideally be within a highvolume unit with sufficient surgeon experience and led by a motivated team of surgeons, nurses, and anaesthetists. The SDD pathway should be clear with a shared and predefined set of stringent criteria regarding patient selection, as well as agreed intra- and postoperative indications for hospital admission. The follow-up schedule should include early face-to-face review, which can be complemented by telephone consultations with the option to expedite emergency assessment as required.

The current evidence for SDD RP remains low due to the retrospective and single centre design of most series, the small number of patients, and the lack of matching.

\section{Conclusions}

SDD RP is a safe and feasible strategy in carefully selected cases and prepared perioperative environment. Our review shows good outcomes with a low risk of complications and readmissions. Optimization of perioperative pathways including ERAS and prehabilitation protocols seems fundamental to increase SDD acceptance and its routine use.
Further research is awaited to confirm cost reductions and potential functional improvements associated with SDD. Prospective and comparative studies assessing patientreported outcomes are needed.

Supplementary Information The online version contains supplementary material available at https://doi.org/10.1007/s00345-022-03944-1.

Author contributions AS, GP: protocol/project development. AS, OE, RGH: data collection or management, AS, IZ, RGH: data analysis. AS, GP, TK, KE, AK, AIAA: manuscript writing/editing.

Funding None.

\section{Declarations}

Conflicts of interest The authors declare that they have no conflict of interest.

Informed consent The authors gave his informed consent for the publication of this study.

\section{References}

1. Palmer JS, Worwag EM, Conrad WG et al (1996) Same day surgery for radical retropubic prostatectomy: is it an attainable goal? Urology 47(1):23-28

2. Ruiz-Deya G, Davis R, Srivastav SK et al (2001) Outpatient radical prostatectomy: impact of standard perineal approach on patient outcome. J Urol 166(2):581-586

3. Ploussard G (2018) Robotic surgery in urology: facts and reality. What are the real advantages of robotic approaches for prostate cancer patients? Curr Opin Urol 28:153-158

4. Martin AD, Nunez RN, Andrews JR et al (2010) Outpatient prostatectomy: too much too soon or just what the patient ordered. Urology 75(2):421-424

5. Kehlet H, Wilmore DW (2002) Multimodal strategies to improve surgical outcome. Am J Surg 183:630-641

6. Ploussard G, Dumonceau O, Thomas L et al (2020) Multi-institutional assessment of routine same day discharge surgery for robot-assisted radical prostatectomy. J Urol. https://doi.org/10. 1097/JU0000000000001129

7. Knoll T, Omar MI, Maclennan S et al (2018) Key steps in conducting systematic reviews for underpinning clinical practice guidelines: methodology of the European Association of Urology. Eur Urol 73:290-300

8. Moga C, Guo B, Schopflocher DHC (2012) Development of a quality appraisal tool for case series. Stud Using a Modif Delphi Tech

9. Wolboldt M, Saltzman B, Tenbrink P et al (2016) Same-day discharge for patients undergoing robot-assisted laparoscopic radical prostatectomy is safe and feasible: results of a pilot study. $\mathrm{J}$ Endourol 30(12):1296-1300

10. Berger AK, Chopra S, Desai MM et al (2016) Outpatient robotic radical prostatectomy: matched-pair comparison with inpatient surgery. J Endourol 30(Suppl 1):S52-S56

11. Abboudi H, Doyle P, Winkler M (2017) Day case laparoscopic radical prostatectomy. Arch Ital Urol Androl 89(3):182-185

12. Banapour P, Elliott P, Jabaji R et al (2019) Safety and feasibility of outpatient robot-assisted radical prostatectomy. J Robot Surg 13(2):261-265 
13. Thomas L, Lacarriere E, Martinache G, Martinache PR (2019) Experience of day case robotic prostatectomy. About thirty-two patients. Prog Urol 29(12):619-626

14. Khalil MI, Bhandari NR, Payakachat N et al (2020) Perioperative mortality and morbidity of outpatient versus inpatient robotassisted radical prostatectomy: a propensity matched analysis. Urol Oncol 38(1):3.e1-3.e6

15. Abaza R, Martinez O, Ferroni MC et al (2019) Same day discharge after robotic radical prostatectomy. J Urol 202(5):959-963

16. Bajpai RR, Razdan S, Barack J et al (2019) Robot-assisted laparoscopic prostatectomy: Is it ready for prime time? A quality of life analysis. J Endourol 33(10):814-822

17. Ploussard G, Almeras C, Beauval JB et al (2020) Same-day discharge surgery for robot-assisted radical prostatectomy in the era of ERAS and prehabilitation pathways: a contemporary, comparative, feasibility study. World J Urol. https://doi.org/10.1007/ s00345-020-03119-w

18. Wilson CA, Aminsharifi A, Sawczyn G et al (2020) Outpatient extraperitoneal single-port robotic radical prostatectomy. Urology 144:142-146

19. Ploussard G, Almeras C, Beauval JB et al (2020) A combination of enhanced recovery after surgery and prehabilitation pathways improves perioperative outcomes and costs for robotic radical prostatectomy. Cancer 126(18):4148-4155

20. Congnard D, Vincendeau S, Lahjaouzi A et al (2019) Outpatient robot-assisted radical prostatectomy: a feasibility study. Urology 128:16-22

21. Dobbs RW, Nguyen TT, Shahait M et al (2020) Outpatient robotassisted radical prostatectomy: Are patients ready for same-day discharge? J Endourol 34(4):450-455
22. Reynolds BR, Bulsara C, Zeps N et al (2018) Exploring pathways towards improving patient experience of robot-assisted radical prostatectomy (RARP): assessing patient satisfaction and attitudes. BJU Int 121:33-39

23. Friðriksson JÖ, Holmberg E, Adolfsson J et al (2014) Rehospitalization after radical prostatectomy in a nationwide, populationbased study. J Urol 192:112-119

24. Ploussard G, Loison G, Almeras C et al (2020) One-day prehabilitation program before robotic radical prostatectomy in daily practice: routine feasibility and benefits for patients and hospitals. Euro Urol Open Sci 21:14-16

25. Patel HR, Cerantola Y, Valerio M et al (2014) Enhanced recovery after surgery: are we ready and can we afford not to implement these pathways for patients undergoing radical cystectomy? Eur Urol 65:263-266

26. Pang KH, Groves R, Venugopal S et al (2017) Prospective implementation of enhanced recovery after surgery protocols to radical cystectomy. Eur Urol 2838(17):30660-30667

27. Kowalsky SJ, Zenati MS, Steve J et al (2019) A Combination of robotic approach and ERAS pathway optimizes outcomes and cost for pancreatoduodenectomy. Ann Surg 269:1138-1145

28. Naresh Row T (2018) Review of day surgery cases at a one day surgery centre. Ambulat Surg 24(3):74-76

Publisher's Note Springer Nature remains neutral with regard to jurisdictional claims in published maps and institutional affiliations. 